Article

\title{
Fault Diagnosis System for Induction Motors by CNN Using Empirical Wavelet Transform
}

\author{
Yu-Min Hsueh, Veeresh Ramesh Ittangihal, Wei-Bin Wu, Hong-Chan Chang \\ and Cheng-Chien Kuo *iD \\ Department of Electrical Engineering, National Taiwan University of Science and Technology, \\ Taipei 10607, Taiwan; D10307009@mail.ntust.edu.tw (Y.-M.H.); valiant.veeru@gmail.com (V.R.I.); \\ andytouchwork@gmail.com (W.-B.W); hcchang@mail.ntust.edu.tw (H.-C.C.) \\ * Correspondence: cckuo@mail.ntust.edu.tw
}

Received: 5 August 2019; Accepted: 27 September 2019; Published: 29 September 2019

\begin{abstract}
Detecting the faults related to the operating condition of induction motors is a very important task for avoiding system failure. In this paper, a novel methodology is demonstrated to detect the working condition of a three-phase induction motor and classify it as a faulty or healthy motor. The electrical current signal data is collected for five different types of fault and one normal operating condition of the induction motors. The first part of the methodology illustrates a pattern recognition technique based on the empirical wavelet transform, to transform the raw current signal into two dimensional (2-D) grayscale images comprising the information related to the faults. Second, a deep CNN (Convolutional Neural Network) model is proposed to automatically extract robust features from the grayscale images to diagnose the faults in the induction motors. The experimental results show that the proposed methodology achieves a competitive accuracy in the fault diagnosis of the induction motors and that it outperformed the traditional statistical and other deep learning methods.
\end{abstract}

Keywords: empirical mode decomposition; pattern recognition; wavelet; empirical wavelet transform; convolutional neural network; induction motor; fourier transform; fault diagnosis

\section{Introduction}

Because of the simple design, low cost, low maintenance, and easy operation, induction motors are one of the most commonly used rotating machines in the industry. In spite of the fact that these machines are more reliable and robust in nature, failure of induction motors is expected, due to the various stresses they encounter during their operating conditions. The most responsible factors behind such failure conditions could be either from mechanical or electrical forces. Different types of machinery faults, like broken bars, bearing faults, an unbalanced rotor, and stator faults and winding faults, have been discussed in the literature [1,2]. Many studies have been conducted on fault diagnosis in recent years. Early detections of the problems are vital to save time and costs, so as to take remedial measures to avoid an entire system failure [3]. The fault diagnosis methods can be classified widely into signal-based, model-based, active/hybrid and knowledge-based methods [4,5]. The knowledge-based methods, also called data-driven methods, require a huge amount of historical data to find the signal patterns for the fault diagnosis of the system.

The predictive maintenance and the data-driven methods are commonly used to analyze signals such as the current, temperature, electrical tension and vibrations, which are captured by the use of sensors $[6,7]$. The signal-based features are extracted for the fault diagnosis. However, the extracted features need to undergo the feature selection techniques to avoid repeated information and also to significantly reduce the feature dimensions, which can improve the performance by retaining important 
features [8]. Finally, the selected features are used for the fault diagnosis via various methods based on traditional statistical and machine learning models. [9-11]. The traditional methods have achieved significant results. However, the feature selection for the methods depends heavily on the knowledge and expertise of signal processing methods with respect to the diagnosis. Furthermore, the traditional machine learning methods are not capable enough to produce distinguishable features of original data and constantly require a process of feature extraction from the signal [10,12-15].

Apart from the significant development in machine learning, deep learning has emerged as an effective study that can overcome the above-mentioned drawbacks in fault diagnosis. It can avoid manual feature extraction and automatically learn the abstract features from the raw data [12]. Various deep learning methods have been studied and applied to fault diagnosis, such as the stacked sparse auto-encoder [13], sparse auto-encoder [15], deep belief network (DBN) [16], denoising auto-encoder [17], and sparse filtering [18]. Deep learning has achieved significant results in comparison to traditional machine learning methods. The convolutional neural network (CNN), known as one of the proven deep learning models, has delivered promising results in learning useful features $[14,19]$. However, there are still many studies to be done on the application of deep learning on fault diagnosis.

In the real world, most of the pattern recognition tasks deal with time-series data. Weather and forecasting, video processing, biomedical signal processing, stock and currency exchange rate data processing have been studied with time-series data [20-22]. Similarly, electrical industry devices such as induction motors also often deal with time-series data like the current, voltage, temperature and vibration signals. Since machinery data signals belong to time-domain signals, a one-dimensional (1-D) CNN is studied to diagnose motor faults [23]. However, in a few cases, the machinery data can be viewed in two dimensions (2-D), such as the time-frequency domain, to avoid redundant data, representing the data as 2-D images using the empirical wavelet transform [24].

As part of this study, the current signals from 3 phase induction motors are considered for a fault diagnosis. All three currents are the same with a difference in the phase, and each phase current was used as one sample for representation in empirical wavelet transform. The main contributions of this work are summarized as follows. First, we propose a well-defined data preprocessing method, in which 2-D features are extracted and represented as an image from the current signal. Second, an effective $\mathrm{CNN}$ model is proposed to extract and learn the features automatically from the images. Finally, the proposed CNN-based method achieves promising results compared to other deep learning and traditional methods.

The remainder of this paper is structured as follows: Section 2 reviews the related works. Section 3 introduces the proposed framework, in which the data preprocessing and proposed CNN model are discussed. Section 4 presents the experimental results. Finally, the conclusion and future research works are presented in Section 5.

\section{Related Works}

In recent years, many signal processing techniques have been studied in the frequency domain, time domain, and time-frequency domain to extract the full features and detect the machine operating condition using classification methods. Time-frequency domain methods are preferred, among others, to analyze and extract the features from the non-stationary signals. Wang et al. [25] applied wavelet scalogram images as an input to CNN to learn the features and detect the faults. Lee et al. [26] analyzed a corrupted raw signal and the effect of the noise on training the CNN model. Ge et al. [27] studied and theoretically analyzed the empirical mode decomposition (EMD) method. Lei et al. [28] used the EMD method to extract features from vibration signals and discussed a kurtosis-based method for fault diagnosis. Pandya et al. [11] constructed an efficient KNN classifier using an asymmetric proximity function for fault diagnosis. Yang et al. [10] proposed an SVM-based method to diagnose the fault patterns of roller bearings. Ngaopitakkul et al. [9] proposed a decision algorithm based on ANN for a fault diagnosis using discrete wavelet transform (DWT) and backpropagation neural networks. The high-frequency component of the current signals is decomposed by using a mother wavelet 
called Daubechies (db4). The DWT extracts the high-frequency component from the fault current signals and the coefficients of the first scale from the DWT are used to detect the fault. Ma et al. [29] proposed a method to extract the features of bearing faults based on the complete ensemble EMD (CEEMD) by enhancing the mode characteristic and via the introduction of adaptive noise to diagnose the bearing faults of rotatory machines. Ge et al. [30] proposed a fault diagnosis method based on an empirical wavelet transform sub modal hypothesis test and ambiguity correlation classification to diagnose the rolling bearing faults using vibration signals. However, the authors concentrated only on rolling bearing faults. Deng et al. [31] studied a fault diagnosis method to extract a new feature by combining Hilbert transform coefficients, the correlation coefficients and the ensemble empirical mode decomposition (EEMD). The vibration signal is decomposed into a list of multiple intrinsic mode functions (IMFs) with distinct frequencies using the EEMD. Agarawal et al. [32] presented a comparative study of ANN and SVM using continuous wavelet transforms and energy entropy methods to diagnose and classify the rolling element bearing faults. Mother base wavelet is selected from four real-valued base wavelets based on the entropy criterions and the energy. The statistical features are extracted from the wavelet coefficients of real signals. The extracted statistical features are provided to ANN and SVM as input for the classification of the bearing faults. These comparative results show SVM giving a better performance than ANN. Jayaswal et al. [33] provided a brief review of recent studies on ANN, fuzzy logic and wavelet transform, used to diagnose rotating machinery faults using raw vibration signals. However, special attention is only given to rolling element bearing faults. Bin et al. [34] studied a method using wavelet coefficients and empirical mode decomposition to extract features and classify faults using a multi-layer perceptron network. However, the ANN study found two main concerns: (1) A large dependency on a prior knowledge of signal processing methods and an expertise in the diagnostic process; and (2) the ANNs studied for the fault diagnosis of induction motors might be limited in their learning capacity from learning complex and nonlinear relationships because of the large information on motor currents. Thus, it is essential to study the deep architecture network for fault diagnosis.

Deep learning is more advanced when compared to traditional machine learning methodology. Due to its potential ways of featuring representation, it has been extensively used in machine health monitoring systems [35]. Jia et al. [36] proposed a neural network-based method to diagnose faults using an auto-encoder. Cho et al. [37] used recurrent neural networks and dynamic Bayesian modeling for fault detection in induction motors. However, with RNN, the information flows via the hidden states and is much slower than with CNN. Deep learning models like deep auto-encoders (DAE), deep belief networks (DBN) and CNNs have been studied for fault diagnosis $[13,14,16]$. Ince et al. [20] used a one-dimensional (1-D) CNN for a real-time motor fault diagnosis. Xu et al. [38] proposed a study based on the Gabor wavelet and the neural network to detect the image intelligence. The authors employed the Gabor wavelet transform to extract the features of information from images. Abdeljaber et al. [39] proposed a 1-D CNN for real-time structural damage detection. Furthermore, there are various ways to represent machinery data in the 2-D format. Chong [40] proposed an effective way to extract the features by converting 1-D vibration signals into 2-D grayscale images. Gaowei et al. [41] proposed a method based on deep $\mathrm{CNN}$ and random forest ensemble learning with a remarkable performance; however, they only focused the bearing fault diagnosis. Lu et al. [42] used a probabilistic neural network as an image classifier by converting signals to images using a bispectrum. Kang et al. [43] used 2-D greyscale images created using Shannon wavelets for an induction motor fault diagnosis. However, an expert's knowledge is necessary for these conversion methods. Although methods such as neural networks, using raw data signals, are considered in many studies in order to diagnose and classify faults, data preprocessing is a highly important action in deep learning. Processing huge quantities of data and examining several qualities of parameters leads to a lot of troubles in data preprocessing. Data with distinct characteristics need distinct methods to extract their characteristics. Many studies use frequency, time-frequency, and histograms to convert signals into images for classification. Similarly, in the proposed study, a two-dimensional matrix generated from wavelet coefficient values is represented 
as an image. The benefit of presenting an image instead of the raw one-dimensional current signal is that the image can provide spatial and temporal dependencies. Moreover, CNN has been a popular deep learning algorithm for working with image datasets, and traditionally it is two dimensional. The benefit of using $\mathrm{CNN}$ over a neural network is its ability to develop an internal representation of a two-dimensional image or a matrix of values. It helps the model to learn the position and scale of different structures in the image data or in the two-dimensional matrix data. It also helps to reduce the number of parameters involved by learning high-level features and via the reusability of weights. In this study, an efficient 1-D signal to 2-D greyscale image representation is proposed by using an empirical wavelet transform. This method is free of any predefined parameters and eliminates the expert's interference.

\section{Proposed Methodology}

This section describes the proposed EWT-CNN-based fault diagnosis methodology. As part of data preprocessing, the raw current signal is converted into images using EWT modes. Then, a deep $\mathrm{CNN}$ model is presented to extract and learn the features for the fault diagnosis.

\subsection{Pattern Recognition Technique}

As the most common data-driven methods are unable to deal with direct original signals for the fault diagnosis, preprocessing the raw signal is necessary. In recent years, an empirical mode decomposition (EMD) algorithm proposed by Huang et al. [44], and has gained a great interest in signal analysis due to its ability to separate stationary and non-stationary components from a signal. However, although its adaptability seems appreciable, the lack of a mathematical theory is the main issue with this approach. To deal with this problem, an Ensemble EMD (EEMD) is proposed to compute several EMD decompositions of the original signal, averaging the decompositions to get a final EEMD. This method seems appreciable, but it increases the computational cost [45].

Currently, wavelet analysis is classified as one of the most used tools to analyze signals. An extensive literature about wavelet theory [46-48] can be referenced for further details. In the temporal domain, with a scaling factor $s>0$ and a translation factor $\mathrm{u} \in \mathbb{R}$, the wavelet dictionary $\left\{\psi_{u, s}\right\}$ is defined as:

$$
\psi_{u, s}(t)=\frac{1}{\sqrt{s}} \psi\left(\frac{t-u}{s}\right)
$$

The scaling factor $\mathrm{s}$ is used to stretch or compress the wavelet function in order to change the oscillating frequency, and the translation facto $u$ is used to change the position of the time window. The wavelet functions define the focal features and time-frequency properties, which can effectively capture the non-stationary characteristics of the signal. There are many wavelets functions that are studied, such as Morlet, Meyer, Symlet, Gabor, Coiflet, and Haar [49-52]. All these methods use either a prescribed scale subdivision or use the output of the classic wavelet output smartly. However, they failed to build a full adaptive wavelet transform. Thus, the proposed method uses a new approach called empirical wavelet transform (EWT) to build a family of wavelets adapted to the processed signal $[24,30]$. The empirical wavelet transform is defined in a step-by-step manner rather than in a single mathematical formulation as is the case of the classic wavelet transform. The main idea behind the EWT is to extract the different modes of a signal based on Fourier supports detected from the spectrum information of the processed signal.

The following steps summarize the empirical wavelet transform proposed in [24]:

Step 1: Find the Fourier transform of the processed input signal.

Step 2: Segment the Fourier spectrum by detecting the local maxima in the spectrum.

Step 3: Sort the local maxima in decreasing order

Step 4: Define the boundaries of every segment as the center between two successive maxima.

Step 5: Follow the construction idea of Meyer's wavelet to obtain a tight frameset

Step 6: Obtain the corresponding signal filters (modes as defined in [24]). 
The proposed empirical wavelets correspond to the dilated version of a single mother wavelet in the temporal domain. However, the corresponding dilatation factors do not follow a prescribed scheme but are detected empirically. For further details on the EWT, we refer the reader to the literature [24]. A three-phase current signal from the induction motor is collected. Ten cycles (one full cycle having 167 data points) for each phase current signal, i.e., 1670 continuous points, are sampled.

Then, the 1670 points are converted into a $1670 \times \mathrm{N}$ time-frequency spectrum, which consists of the coefficient matrices via the empirical wavelet transform. $\mathrm{N}$ stands for the number of modes, and the sufficient raw signal characteristics can be obtained by choosing the appropriate value. Finally, the grayscale image is represented from the time-frequency spectrum.

The raw current signals collected from the different induction motors working at different faulty/healthy condition and operating on the same load condition are shown in Figure 1. However, they are non-distinguishable, and it is almost impossible to diagnose the fault condition of the motors by using the raw current signals. Figure 2 shows the same set of raw signals that are processed by EWT, and they look absolutely distinguishable from each other. Hence, it is indeed necessary to preprocess the raw current signals by EWT in order to find the distinguishable patterns.
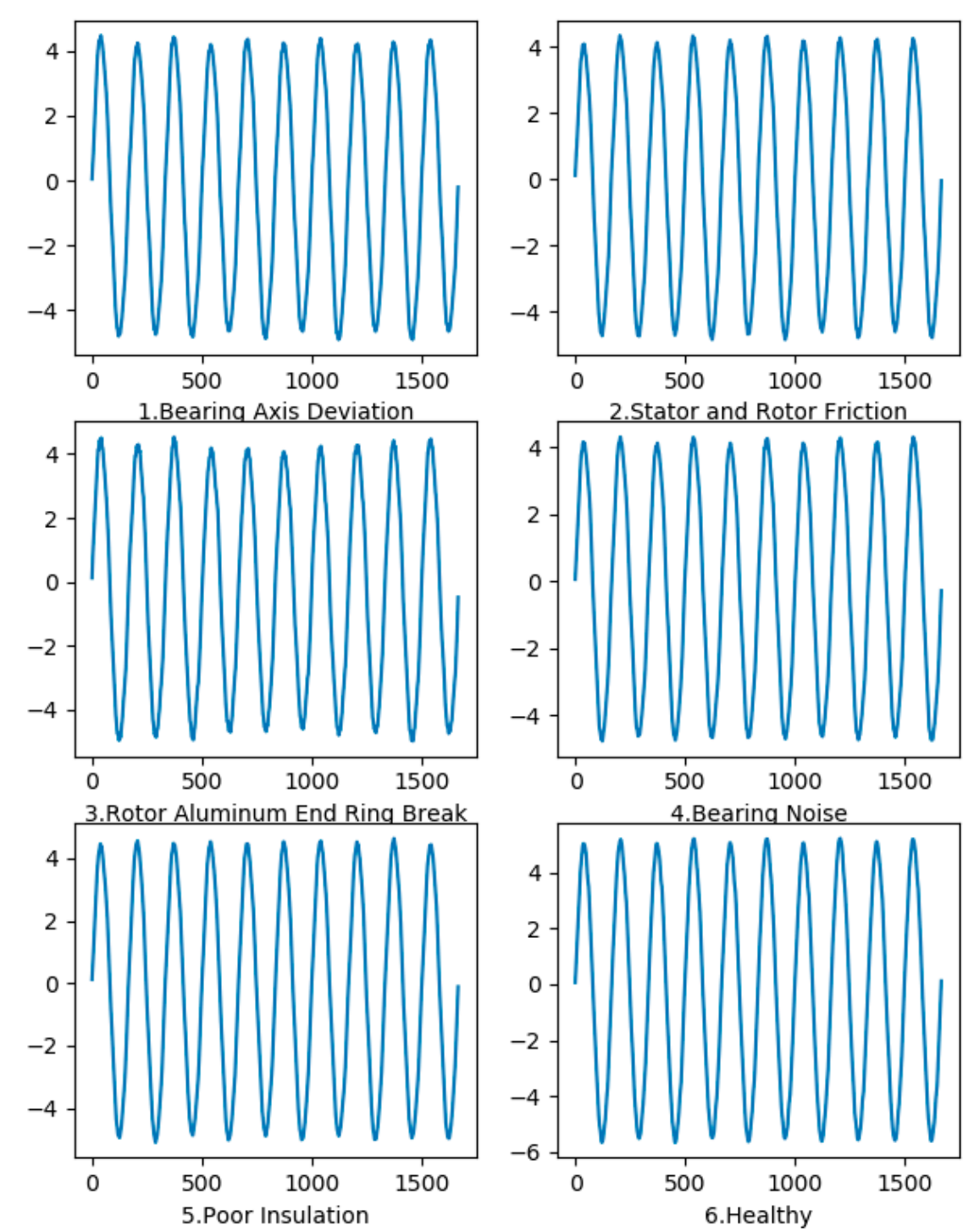

Figure 1. The induction motor current signals: 10 cycles of current signals for each fault and healthy conditions motors (non-distinguishable patterns). 

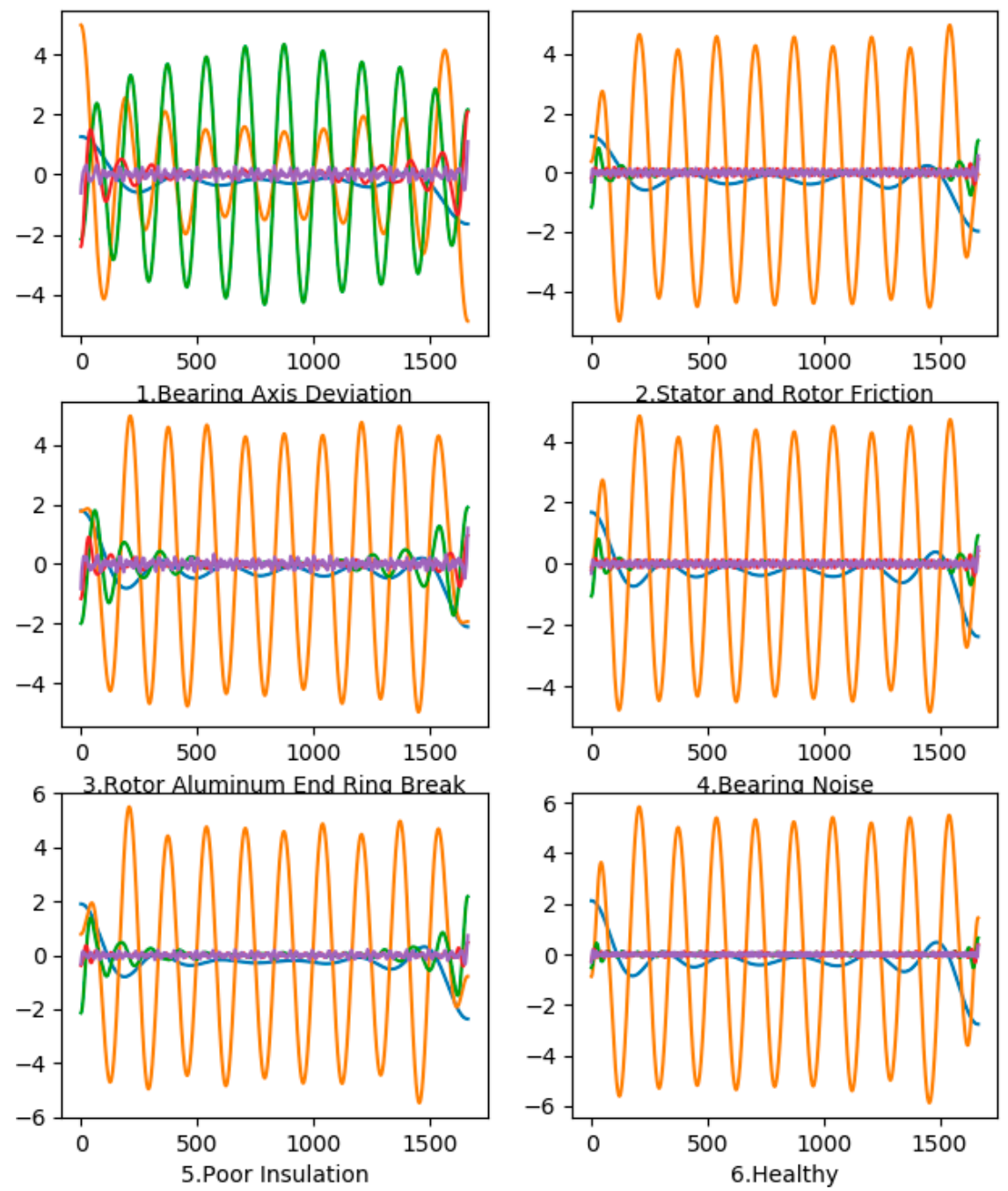

Figure 2. The EWT modes of the current signals: The EWT modes plot for the respective fault and healthy conditions (distinguishable patterns).

The CNN model training will be difficult with the $1670 \times \mathrm{N}$ image, as the latter results in computational complexity. A simple image resizing method based on scikit-image processing [53] is used to decrease the image size. Figure 3 illustrates the entire workflow of the proposed method. Figure 4 shows the distinguishable grayscale resized $(32 \times 32)$ images for each fault type and the healthy type of motors data.

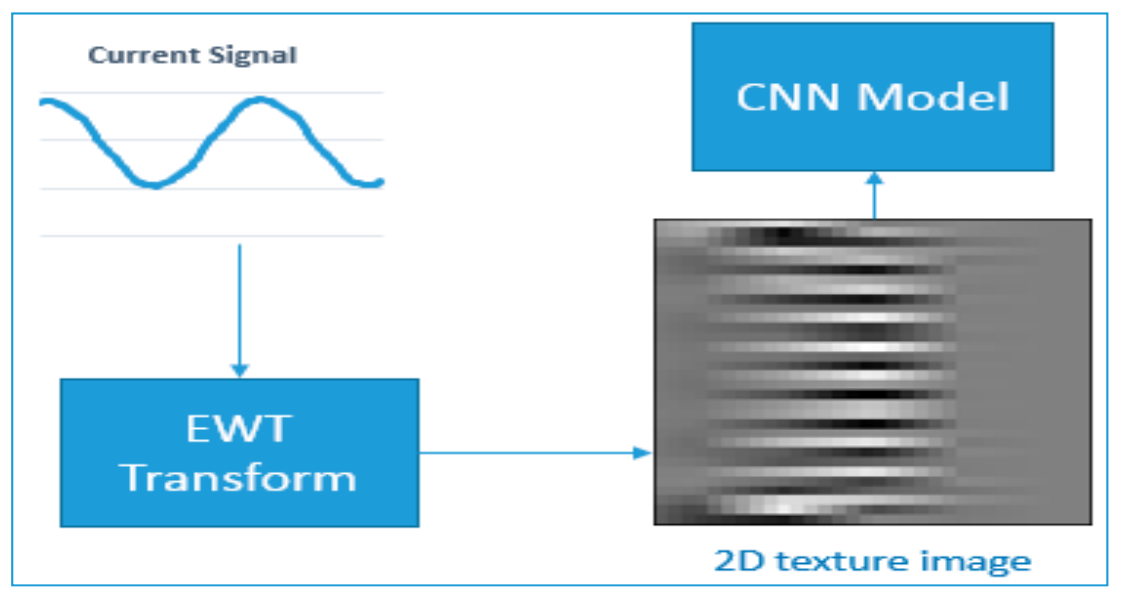

Figure 3. The architecture of the proposed methodology. 

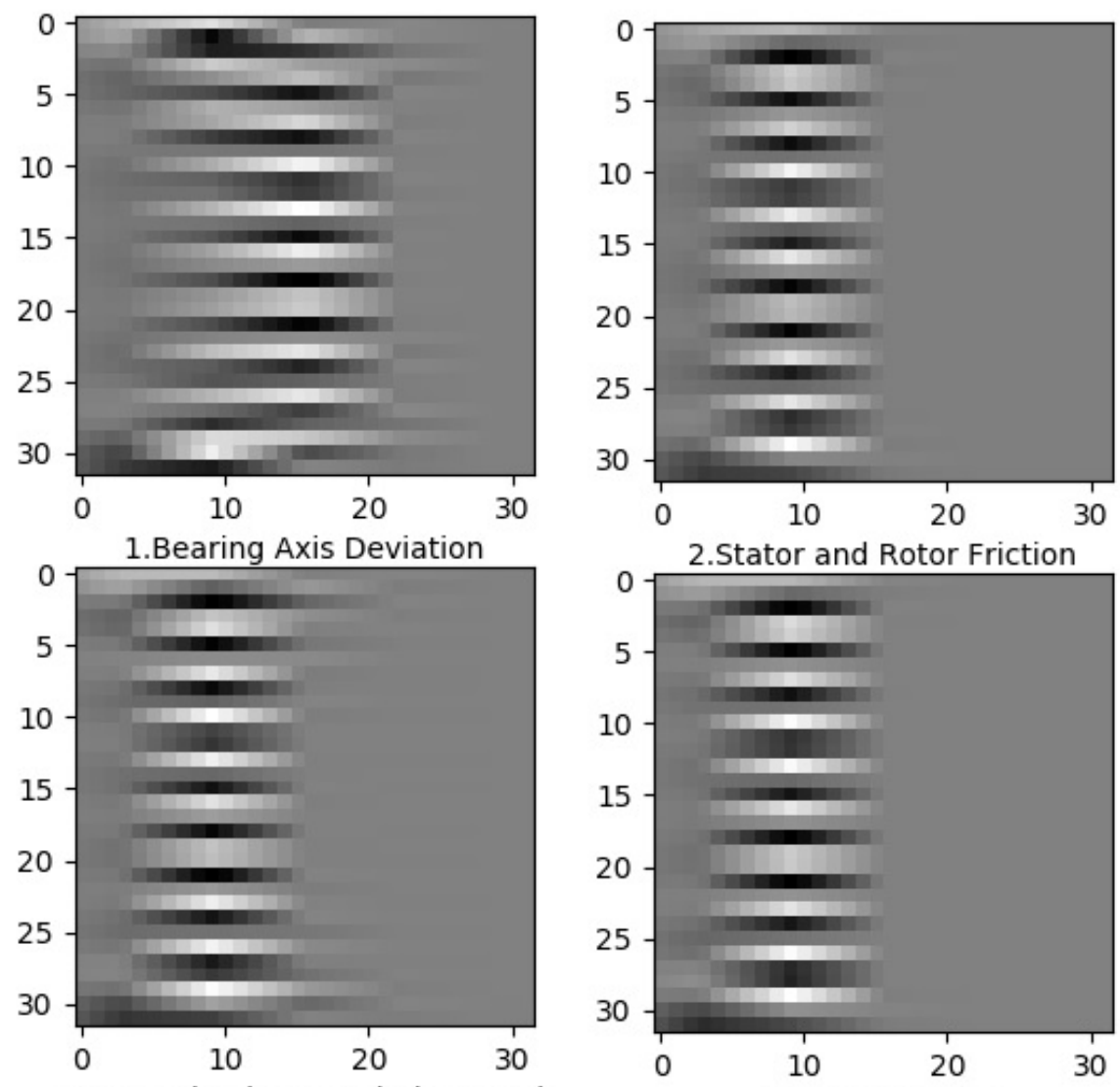

3.Rotor Aluminum End Ring Break
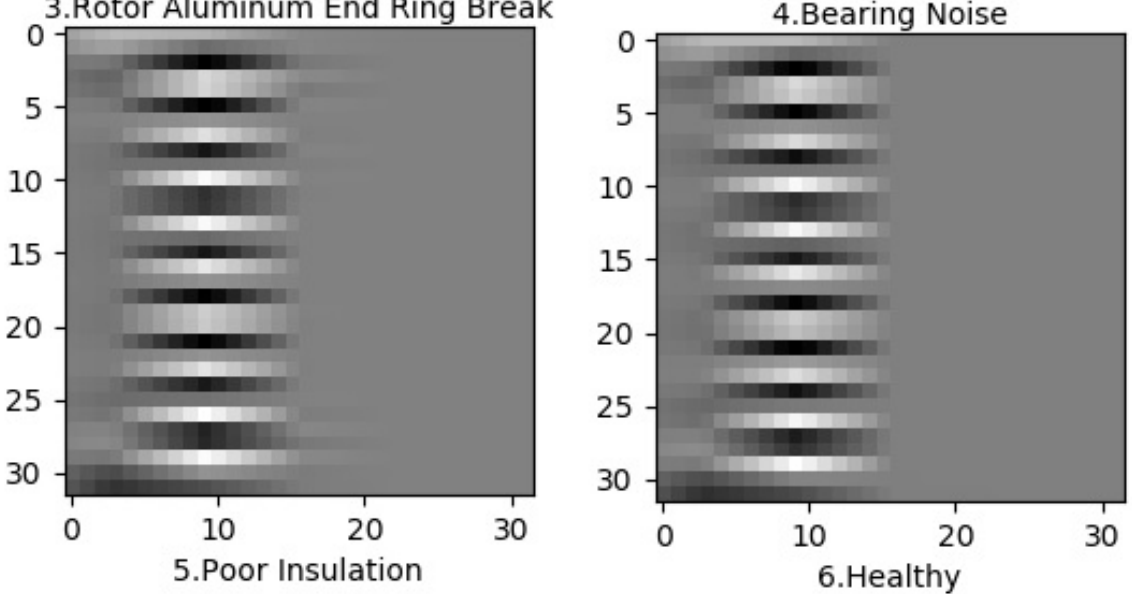

Figure 4. Grayscale images $(32 \times 32)$ for the EWT modes of each fault and the healthy conditions of the motor.

\subsection{Proposed Deep Convolutional Neural Network}

After converting the raw current signals into grayscale images, a deep CNN model is designed and pre-trained for feature learning. The proposed deep $\mathrm{CNN}$ has a three-stage structure. Each stage represents a feature learning stage with a different feature-level, which includes convolution, activation, and pooling layers.

Figure 5 illustrates the architecture of the proposed CNN model, which consists of three convolutional layers with filters $32-3 \times 3,64-3 \times 3$ and $128-3 \times 3$, respectively. In addition to that, there are three max-pooling layers of size $2 \times 2$. The most commonly-used activation functions are the hyperbolic tangent, softmax, ReLU, and sigmoid function [54]. Among them, ReLU has proven to be more effective than the others. However, during the training, ReLU units can die, and this could occur when a large gradient flows through a ReLU neuron. This causes the weights to update, so 
that the neuron will never activate again on any data point. A leaky ReLU is an attempt to solve this problem [55,56]; thus, the leaky ReLU (Rectified Linear Units) is applied as an activation function to introduce non-linearity into each stage, allowing the CNN to learn complex models. Pooling is used to reduce the resolution of the input image via the process of subsampling, and Max Pooling is used in the proposed model.

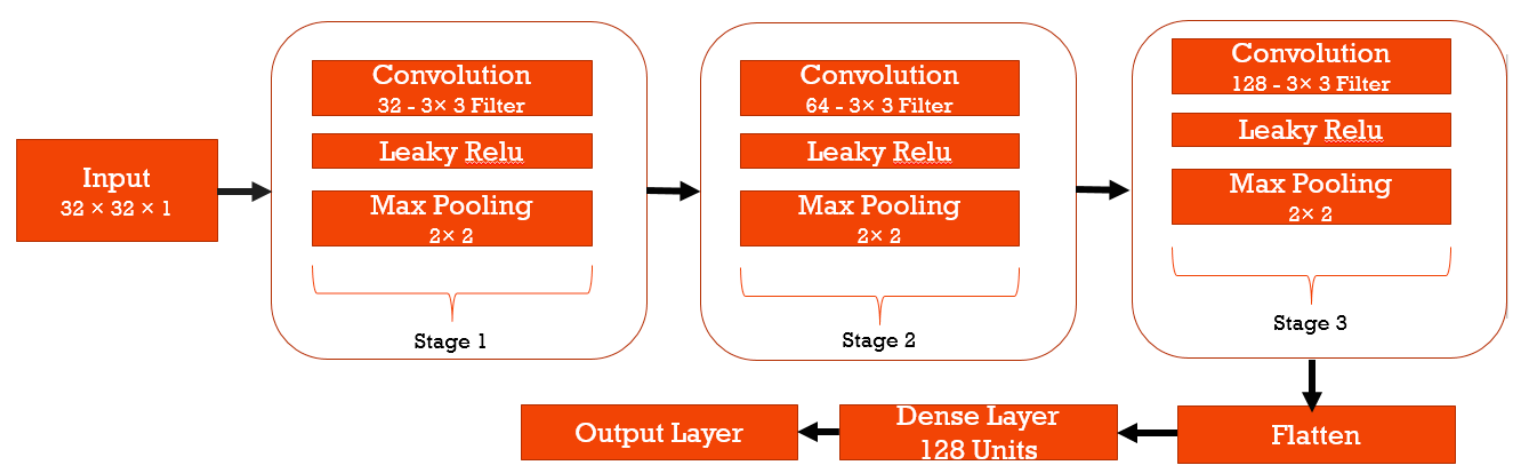

Figure 5. The proposed 3-stage Convolutional Neural Network Architecture diagram. $32 \times 32$ size grayscale images are fed into the CNN model. The architecture consists of three convolutions layers followed by pooling layers and two fully connected layers.

Training the CNN model involves learning all the weights and biases, and it is important to optimize these parameters for an efficient feature learning. Apart from the training parameters, the CNN also needs to optimize the hyperparameters, such as the learning rate and dropout. The dropout is an important property of $\mathrm{CNN}$, which can greatly help in preventing the overfitting by generalizing the model [57]. A dropout of size 0.4 is used for a better regularization in the proposed CNN. The adapted moment estimation (ADAM), which is a backpropagation algorithm, is used to optimize the learning rate and other hyperparameters. The ADAM adapts the learning rate scale through different layers and avoids the manual assignment for choosing the best learning rate [58]. At the end of the three stages, the feature maps are flattened and classified via a fully connected layer for 6 types of classifications.

\section{Experimental Results and Discussion}

To assess the performance of the proposed methodology, the raw current signal data from an experimental setup involving a total of six induction motors with the same specifications are used. This includes one healthy and five fault types of raw current data signals, which are collected from the experimental setup. The six types of current signals are studied and analyzed for the healthy condition of the motor, as well as for the following five faulty conditions of the motor [59]. The data preprocessing and the CNN model are written in Python 3.6 with TensorFlow and run on the Windows 64 bit operating system.

\subsection{Faults in Induction Motors}

The motors undergo various types of failure modes, mostly due to electrical and mechanical forces. These failure modes eventually break the entire system from its normal working condition. This section deals mainly with the five types of faults, namely: bearing axis deviation, stator and rotor friction, rotor aluminum end ring break, bearing noise and poor insulation.

1. Bearing Axis Deviation: The structure of the bearing is precise. If it is disturbed by some external forces, the structure of the bearing may be affected. After connecting the motor to the load, an earthquake, collision, and the assembly process may introduce an offset of midpoints on both ends of the connection, which causes heating problems and unwanted noise. A normal motor with a full load is used, and, for this experiment, the coupling is shifted $0.5 \mathrm{~mm}$ upward to imitate the deviation condition. The experimental motor model is shown in Figure 6d. 
2. Stator and Rotor Friction and Poor Insulation: Because of friction, overheating, insulation aging, dampness and corona, the stator or rotor coil is short-circuited, and hence it will break down if not diagnosed. The insulation of the adjacent turns in the stator coil will be damaged, causing a short circuit, as shown in the Figure 6a. When the motor is started, the short-circuit current value will be high due to the difference in excessive voltage caused by different wound turns in the stator, and the motor will be burnt. The experimental motor model is shown in Figure 6a.

3. Rotor Aluminum End Ring Break: The outer ring damage is one of the most common faults. If the starting frequency is very high and/or the motor is overloaded, the rotor bar will break due to the excessive current. For this experiment, a hole with a diameter of $7 \mathrm{~mm}$ and a depth of $30 \mathrm{~mm}$ is made on the rotor bar to simulate the fault condition. The experimental motor model is shown in Figure $6 b$.

4. Bearing Noise: Damage to the bearing's outer race is considered one of the constant faults observed in bearings. The structure of the bearing is always kept precise. However, if the structure is disturbed by an external force or some other structures of bearing, this causes messy and numerous harmonics in the measured spectrum. A hole with a diameter and depth of $1 \mathrm{~mm}$ is made in the outer race to simulate the fault condition for this experiment. The experimental motor model is shown in Figure 6c.

The proposed method uses the motor raw current signal values to analyze and find patterns for the fault diagnosis of the above-listed motor faults.

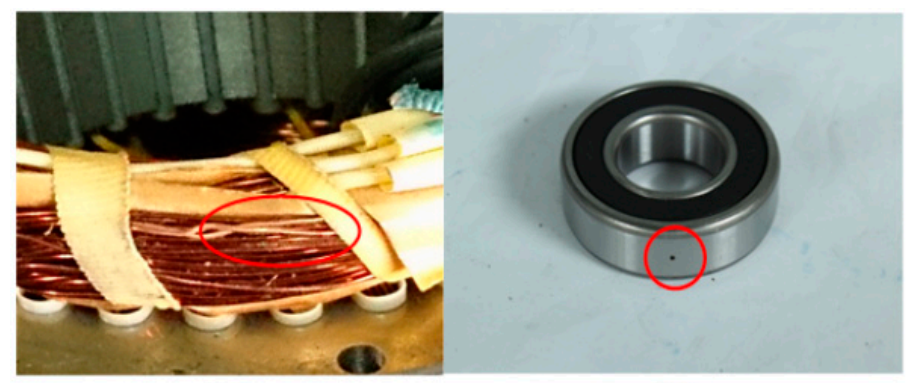

(a)

(c)

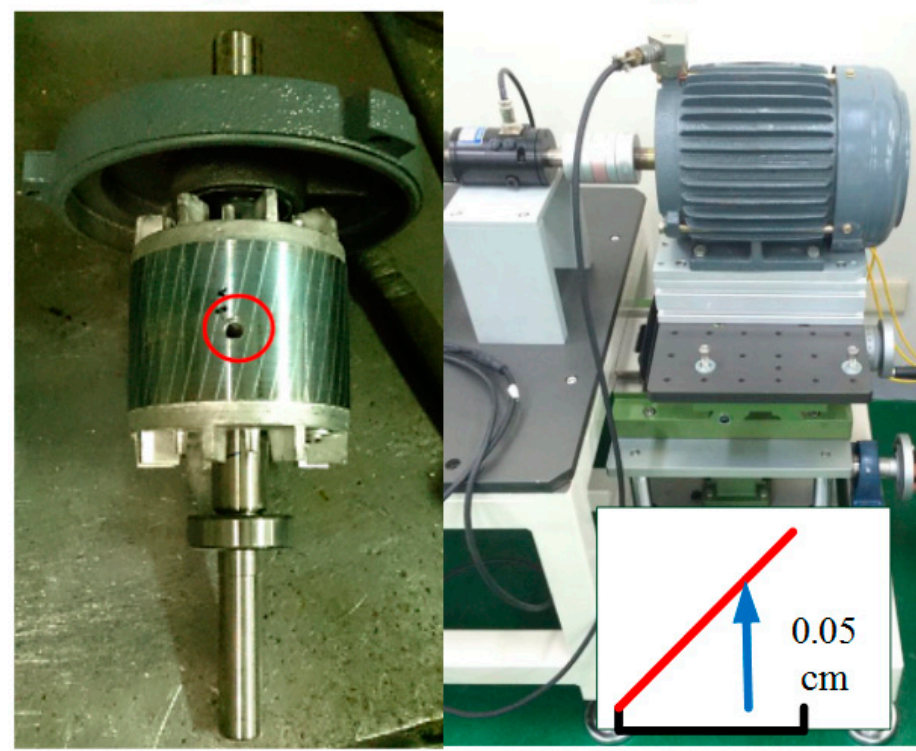

(b)

(d)

Figure 6. The experimental motor model: (a) stator and rotor friction and poor insulation; (b) rotor aluminum end ring break; (c) bearing noise; and (d) bearing axis deviation. 


\subsection{Dataset}

The collected dataset from the experiment consists of 900 samples [60]. 50 samples from the healthy condition motor and 50 samples from each kind of faulty condition motor on a $100 \%$ load (full load) are collected and analyzed. As three-phase induction motors are used in this study, there are three current signals, with differences in the phase, and each phase current is considered when preparing the dataset. Hence, a total of 150 raw current data samples are prepared for the healthy motor and for each of the five faulty motors, as described in Table 1.

Table 1. The dataset samples used for the evaluation.

\begin{tabular}{ccccccc}
\hline $\begin{array}{c}\text { Bearing Axis } \\
\text { Deviation }\end{array}$ & $\begin{array}{c}\text { Stator and } \\
\text { Rotor Friction }\end{array}$ & $\begin{array}{c}\text { Rotor Aluminum } \\
\text { End Ring Break }\end{array}$ & $\begin{array}{c}\text { Bearing } \\
\text { Noise }\end{array}$ & $\begin{array}{c}\text { Poor } \\
\text { Insulation }\end{array}$ & Healthy & Total \\
\hline 150 & 150 & 150 & 150 & 150 & 150 & 900 \\
\hline
\end{tabular}

The data set is divided into three parts, as described in Table 2. $70 \%$ of the dataset (630 samples for training) and $15 \%$ of the dataset (135 samples for validation) are used simultaneously to train the CNN model. The remaining 15\% (135 image samples) are used to test the trained CNN model. Cross-validation techniques are often used for simple models having few trainable parameters like linear regression, logistic regression, small neural networks and support vector machines. A CNN model having many parameters will lead to too many possible changes in the architecture. However, in this study, the proposed CNN model is trained and evaluated using a k-fold cross-validation with the data split ratio shown in Table 2.

Table 2. The dataset samples used for the evaluation.

\begin{tabular}{ccc}
\hline \multicolumn{3}{c}{ Data Split Ratio } \\
\hline Training & $70 \%$ & 630 \\
Validation & $15 \%$ & 135 \\
Test & $15 \%$ & 135 \\
\hline
\end{tabular}

\subsection{CNN Performance Evaluation Results}

The proposed CNN model is trained over 150 epochs to learn the robust features for each type of faulty condition motor and one normal operating condition motor. A $\mathrm{k}$-fold cross-validation technique with five folds is applied manually to evaluate the model training and testing. The CNN model is trained to extract and learn the features from 630 samples of the training dataset, simultaneously validated against 135 samples of the validation dataset during each iteration for the five folds of the dataset split. The trained CNN model is evaluated against 135 samples of the test-dataset. The model is cross-validated over five folds with the dataset split ratio being described in Table 2, after which the averages of all the accuracies and losses during each fold are collected in order to observe the accuracies and losses during the training, as shown in Figure 7.

The proposed CNN model is trained and tested with batch sizes of 16, 32 and 64, and we found the best results to be with a size of 32 . The CNN model is trained over 50 to 200 epochs to learn the robust features and analyze the classification performance, in order to choose the number of epochs. The average accuracies and losses (training and validation) are collected at each iteration while training the CNN model with a k-fold cross-validation technique and are then plotted, as shown in Figure 7. The CNN model hit the training accuracy by almost $100 \%$ with a validation accuracy of around $91 \%$. Over the 150 epochs, the proposed CNN model was able to learn the robust and generalized features of the EWT grayscale images, in order to diagnose the motor faults and classify them into faulty or healthy categories. 

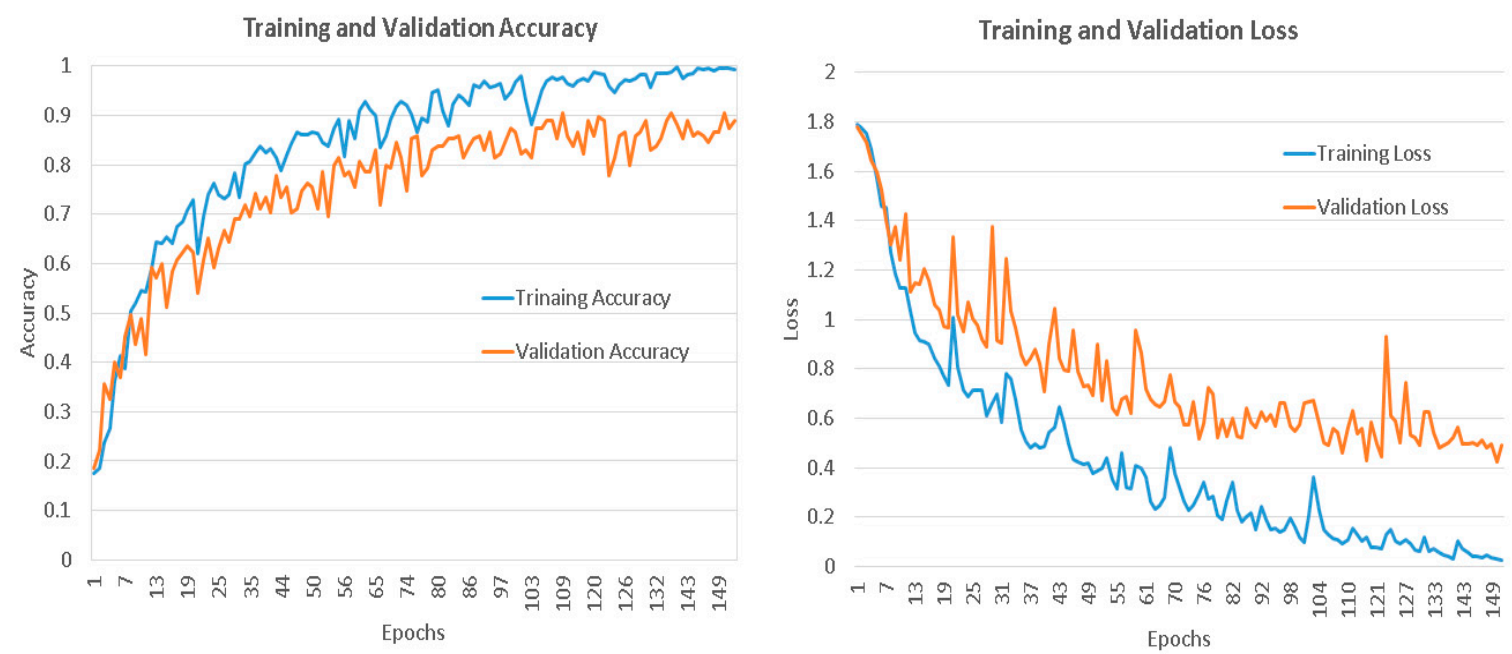

Figure 7. The accuracy and loss curves over 150 epochs of the CNN training.

To evaluate the performance of the trained CNN model, 135 samples of the test dataset are used. The performance result of the trained CNN model looks interesting, with an average accuracy of $97 \%$ on the test dataset, as described in the classification report (Table 3). From the classification report, it can be clearly seen that the proposed CNN model is capable of extracting and learning the features from the test dataset and of classifying the features for the respective faulty and healthy conditions. The proposed model is able to classify the healthy condition, bearing axis deviation fault, rotor aluminum end ring break fault and bearing noise fault more effectively than the other faults. However, the model needs to be tuned in the case of the motor with poor insulation faulty condition. Poor insulation can also be observed due to stator and rotor friction and bearing axis deviation. Hence, there are some misclassifications with other types. Figure 8 illustrates the confusion matrix, which explains the classification results on the test dataset (135 samples) using the well-trained CNN model. Almost all the test samples are correctly classified, with a few misclassifications involving the poor insulation condition and other faulty conditions.

\begin{tabular}{|c|l|c|c|c|c|c|c|c|}
\hline & \multicolumn{7}{|c|}{ Confusion Matrix } \\
\cline { 2 - 10 } & class & Fault 0 & Fault 1 & Fault 2 & Fault 3 & Fault 4 & Fault 5 & \\
\cline { 2 - 10 } & Fault 0 & $\mathbf{2 3}$ & 0 & 0 & 0 & 0 & 0 & 23 \\
\cline { 2 - 10 } & Fault 1 & 0 & $\mathbf{2 2}$ & 0 & 0 & 0 & 0 & 22 \\
\cline { 2 - 10 } & Fault 2 & 0 & 0 & $\mathbf{2 1}$ & 0 & 0 & 1 & 22 \\
\cline { 2 - 10 } & Fault 3 & 0 & 0 & 0 & $\mathbf{2 3}$ & 0 & 1 & 24 \\
\hline & Fault 4 & 0 & 0 & 1 & 0 & $\mathbf{2 1}$ & 0 & 22 \\
\hline & Fault 5 & 0 & 0 & 0 & 0 & 1 & $\mathbf{2 1}$ & 22 \\
\hline & 23 & 22 & 22 & 23 & 22 & 23 & 135 \\
\hline
\end{tabular}

Figure 8. The confusion matrix for the test dataset. (Fault 0: Healthy, Fault 1: Bearing Axis Deviation, Fault 2: Stator and Rotor Friction, Fault 3: Rotor Aluminum End Ring Break, Fault 4: Bearing Noise, Fault 5: Poor Insulation).

In order to assess the performance metrics of the proposed deep CNN model, a few of the other statistical and deep learning models are chosen to compare them with the proposed deep CNN model. The experiment of comparing this model with the traditional methodologies is conducted with the same dataset that was considered to evaluate the proposed model. The collected dataset is used to 
evaluate the traditional and other deep learning models listed in Table 4. The proposed methodology is compared with the deep belief network (DBN) [16], SVM [61], sparse filter [18], ANN [16] and adaptive deep convolutional neural network (ADCNN) [62]. Similar to the proposed CNN model, a k-fold cross-validation technique with five folds is used to train these methods. The test data (135) samples are used to evaluate these models. The prediction accuracy for the test dataset is collected for each of these methods and presented in Table 4.

Table 3. The classification report of the test dataset.

\begin{tabular}{ccccc}
\hline \multicolumn{5}{c}{ Classification Report } \\
\hline CLASS & Precision & Recall & F1-Score & Support \\
\hline Healthy & 1.00 & 1.00 & 1.00 & 23 \\
\hline Bearing Axis Deviation & 0.97 & 0.96 & 0.94 & 22 \\
\hline Stator and Rotor Friction & 0.95 & 0.97 & 0.96 & 22 \\
\hline Rotor Aluminum End Ring Break & 1.00 & 1.00 & 1.00 & 23 \\
\hline Bearing Noise & 0.93 & 1.00 & 0.96 & 22 \\
\hline Poor Insulation & 0.96 & 0.89 & 0.95 & 23 \\
\hline Accuracy & & & 0.97 & 135 \\
\hline Macro avg & 0.97 & 0.97 & 0.97 & 135 \\
\hline Weighted avg & 0.97 & 0.97 & 0.97 & 135 \\
\hline
\end{tabular}

Table 4. The comparison results.

\begin{tabular}{cc}
\hline Methods & Accuracy (\%) \\
\hline DBN & 92.2 \\
\hline SVM & 89.8 \\
\hline Sparse filter & 96.4 \\
\hline ANN & 81.8 \\
\hline ADCNN & 96.2 \\
\hline Proposed CNN & $\mathbf{9 7 . 3 7}$ \\
\hline
\end{tabular}

The comparison results explain that the proposed deep CNN model attains a prominent result when compared to the other methods. The prediction accuracy is $97.37 \%$, which is better than all the other methods; this shows the significant performance of the proposed deep CNN model.

\section{Conclusions and Future Work}

An effective methodology was presented to diagnose the faults in a three-phase induction motor based on EWT and deep CNN. The main contributions of this study are that we propose a method to convert time-series data, such as current signals, into grayscale images, using EWT and applying the proposed deep CNN model to classify the EWT grayscale images for a fault diagnosis. The proposed methodology was tested for five fault types of the induction motor, including bearing axis deviation, stator and rotor friction, rotor aluminum end ring break, bearing noise and poor insulation, and it achieved a significant accuracy of $97.37 \%$. The proposed methodology performed better than the other traditional and deep learning methods. We demonstrated that the proposed methodology, which took into account a single variable as the input feature, yielded promising results when compared to rule-based diagnosis systems that take into account multiple features for a fault diagnosis.

The limitations of the proposed methodology are as follows. First, the dataset that was considered for the experiment was comparatively small, and a huge number of data samples need to be collected 
for different load conditions, such as no load, half load or full load. Second, data from motors with different specifications need to be collected in order to learn more generalized features. Third, the most common faults in induction motors need to be detected in order to avoid misclassifications. Based on the limitations described above, our future work is focused on collecting more data samples from induction motors having different specifications and working at different loads, as well as investing in collecting information on the most common fault types in induction motors in order to avoid misclassification. Furthermore, $\mathrm{CNN}$-based transfer learning can be studied to reduce training costs.

Author Contributions: Y.-M.H. has generated the data and analyzed the faults in induction motors. W.-B.W. validated the data for each kind of fault. V.R.I. performed data preprocessing to train the CNN model and evaluated the trained model for fault diagnosis. Y.-M.H. and V.R.I. analyzed the experimental results with guidance from C.-C.K.; C.-C.K. and H.-C.C. revised the manuscript for submission.

Funding: This research received no external funding.

Conflicts of Interest: The authors declare no conflict of interest.

\section{References}

1. Albrecht, P.F.; Appiarius, J.C.; McCoy, R.M.; Owen, E.L.; Sharma, D.K. Assessment of the Reliability of Motors in Utility Applications-Updated. IEEE Trans. Energy Convers. 1986, EC-1, 39-46. [CrossRef]

2. Bonnett, A.H.; Soukup, G.C. Cause and Analysis of Stator and Rotor Failures in Three-Phase Squirrel-Cage Induction Motors. IEEE Trans. Ind. Appl. 1992, 28, 921-937. [CrossRef]

3. Dai, X.; Gao, Z. From model signal to knowledge: A data-driven perspective of fault detection and diagnosis. IEEE Trans. Ind. Inform. 2013, 9, 2226-2238. [CrossRef]

4. Gao, Z.; Cecati, C.; Ding, S.X. A survey of fault diagnosis and fault-tolerant techniques-Part I: Fault diagnosis with model-based and signal-based approaches. IEEE Trans. Ind. Electron. 2015, 62, 3757-3767. [CrossRef]

5. Cecati, C. A survey of fault diagnosis and fault-tolerant techniques-Part II: Fault diagnosis with knowledge-based and hybrid/active approaches. IEEE Trans. Ind. Electron. 2015, 62, 3768-3774.

6. Lee, J.; Wu, F.; Zhao, W.; Ghaffari, M.; Liao, L.; Siegel, D. Prognostics and health management design for rotary machinery systems-Reviews, methodology and applications. Mech. Syst. Signal Process. 2014, 42, 314-334. [CrossRef]

7. Lamim Filho, P.C.M.; Pederiva, R.; Brito, J.N. Detection of stator winding faults in induction machines using flux and vibration analysis. Mech. Syst. Signal Process. 2014, 42, 377-387. [CrossRef]

8. Sun, W.; Chen, J.; Li, J. Decision tree and PCA-based fault diagnosis of rotating machinery. Noise Vib. Worldw. 2007, 21, 1300-1317. [CrossRef]

9. Ngaopitakkul, A.; Bunjongjit, S. An application of a discrete wavelet transform and a back-propagation neural network algorithm for fault diagnosis on single-circuit transmission line. Int. J. Syst. Sci. 2013, 44, 1745-1761. [CrossRef]

10. Yang, Y.; Yu, D.; Cheng, J. A fault diagnosis approach for roller bearing based on IMF envelope spectrum and SVM. Measurement 2007, 40, 943-950. [CrossRef]

11. Pandya, D.H.; Upadhyay, S.H.; Harsha, S.P. Fault diagnosis of rolling element bearing with intrinsic mode function of acoustic emission data using APF-KNN. Expert Syst. Appl. 2013, 40, 4137-4145. [CrossRef]

12. Lecun, Y.; Bengio, Y.; Hinton, G. Deep learning. Nature 2015, 521, 436-444. [CrossRef] [PubMed]

13. Qi, Y.; Shen, C.; Wang, D.; Shi, J.; Jiang, X.; Zhu, Z. Stacked Sparse Autoencoder-Based Deep Network for Fault Diagnosis of Rotating Machinery. IEEE Access 2017, 5, 15066-15079. [CrossRef]

14. Xia, M.; Li, T.; Xu, L.; Liu, L.; Silva, C.W. Fault Diagnosis for Rotating Machinery Using Multiple Sensors and Convolutional Neural Networks. IEEE/ASME Trans. Mechatron. 2018, 23, 101-110. [CrossRef]

15. Wen, L.; Gao, L.; Li, X. A New Deep Transfer Learning Based on Sparse Auto-Encoder for Fault Diagnosis. IEEE Trans. Syst. Man Cybern. Syst. 2017, 99, 1-9. [CrossRef]

16. Shao, H.; Jiang, H.; Zhang, X.; Niu, M. Rolling bearing fault diagnosis using an optimization deep belief network. Meas. Sci. Technol. 2015, 26, 115002. [CrossRef]

17. Shao, H.; Jiang, H.; Wang, F.; Zhao, H. An enhancement deep feature fusion method for rotating machinery fault diagnosis. Knowl. Based Syst. 2017, 119, 200-220. [CrossRef] 
18. Lei, Y.; Jia, F.; Lin, J.; Xing, S.; Ding, S.X. An intelligent fault diagnosis method using unsupervised feature learning towards mechanical big data. IEEE Trans. Ind. Electron. 2016, 63, 3137-3147. [CrossRef]

19. Lee, K.B.; Cheon, S.; Chang, O.K. A Convolutional Neural Network for Fault Classification and Diagnosis in Semiconductor Manufacturing Processes. IEEE Trans. Semicond. Manuf. 2017, 30, 135-142. [CrossRef]

20. Wang, J.; Liu, P.; She, M.; Nahavandi, S.; Kouzani, A. Bag-of-words representation for biomedical time series classification. Biomed. Signal Process. Control 2016, 8, 634-644. [CrossRef]

21. Hatami, N.; Chira, C. Classifiers with a reject option for early time-series classification. In Proceedings of the 2013 IEEE Symposium on Computational Intelligence and Ensemble Learning (CIEL), Singapore, 16-19 April 2013; pp. 9-16.

22. Wang, Z.; Oates, T. Pooling sax-bop approaches with boosting to classify multivariate synchronous physio-logical time series data. In Proceedings of the 28th International FLAIRS Conference, Hollywood, FL, USA, 18-20 May 2015; pp. 335-341.

23. Ince, T.; Kiranyaz, S.; Eren, L.; Askar, M.; Gabbouj, M. Real-time motor fault detection by 1-D convolutional neural networks. IEEE Trans. Ind. Electron. 2016, 63, 7067-7075. [CrossRef]

24. Jerome, G. Empirical Wavelet Transform. IEEE Trans. Signal Process. 2013, 61, 3999-4010.

25. Wang, J.; Zhuang, J.; Duan, L.; Cheng, W. A multi-scale convolution neural network for featureless fault diagnosis. In Proceedings of the International Symposium on Flexible Automation (ISFA'16), Cleveland, OH, USA, 1-3 August 2016.

26. Lee, D.; Siu, V.; Cruz, R.; Yetman, C. Convolutional neural net and bearing fault analysis. In Proceedings of the International Conference on Data Mining Series (ICDM) Barcelona, San Diego, CA, USA, 12-15 December 2016; pp. 194-200.

27. Ge, H.; Chen, G.; Yu, H.; Chen, H.; An, F. Theoretical Analysis of Empirical Mode Decomposition. Symmetry 2018, 10, 623. [CrossRef]

28. Lei, Y.; He, Z.; Zi, Y. EEMD method and WNN for fault diagnosis of locomotive roller bearings. Expert Syst. Appl. 2011, 38, 7334-7341. [CrossRef]

29. Ma, F.; Zhan, L.; Li, C.; Li, Z.; Wang, T. Self-Adaptive Fault Feature Extraction of Rolling Bearings Based on Enhancing Mode Characteristic of Complete Ensemble Empirical Mode Decomposition with Adaptive Noise. Symmetry 2019, 11, 513. [CrossRef]

30. Ge, M.; Wang, J.; Xu, Y.; Zhang, F.; Bai, K.; Ren, X. Rolling Bearing Fault Diagnosis Based on EWT Sub-Modal Hypothesis Test and Ambiguity Correlation Classification. Symmetry 2018, 10, 730. [CrossRef]

31. Deng, W.; Zhao, H.; Yang, X.; Dong, C. A Fault Feature Extraction Method for Motor Bearing and Transmission Analysis. Symmetry 2017, 9, 60. [CrossRef]

32. Agrawal, P.; Jayaswal, P. Diagnosis and Classifications of Bearing Faults Using Artificial Neural Network and Support Vector Machine. J. Inst. Eng. India Ser. C 2019, 1-12. [CrossRef]

33. Jayaswal, P.; Wadhwani, A.K. Application of artificial neural networks, fuzzy logic and wavelet transform in fault diagnosis via vibration signal analysis: A review. Aust. J. Mech. Eng. 2015, 7, 157-171. [CrossRef]

34. Bin, G.F.; Gao, J.J.; Li, X.J.; Dhillon, B.S. Early fault diagnosis of rotating machinery based on wavelet packets-Empirical mode decomposition feature extraction and neural network. Mech. Syst. Signal Process. 2012, 27, 696-711. [CrossRef]

35. Zhao, R.; Yan, R.; Chen, Z.; Mao, K.; Wang, P.; Gao, R.X. Deep learning and its applications to machine health monitoring: A survey. arXiv 2016, arXiv:1612.07640. [CrossRef]

36. Jia, F.; Lei, Y.; Lin, J.; Zhou, X.; Lu, N. Deep neural networks: A promising tool for fault characteristic mining and intelligent diagnosis of rotating machinery with massive data. Mech. Syst. Signal Process. 2016, 72, 303-315. [CrossRef]

37. Cho, H.C.; Knowles, J.; Fadali, M.S.; Lee, K.S. Fault detection and isolation of induction motors using recurrent neural networks and dynamic Bayesian modeling. IEEE Trans. Control Syst. Technol. 2009, 18, 430-437. [CrossRef]

38. Xu, Y.; Liang, F.; Zhang, G.; Xu, H. Image Intelligent Detection Based on the Gabor Wavelet and the Neural Network. Symmetry 2016, 8, 130. [CrossRef]

39. OAbdeljaber; Avci, O.; Kiranyaz, S.; Gabbouj, M.; Inman, D.J. Real-time vibration-based structural damage detection using one-dimensional convolutional neural networks. J. Sound Vib. 2017, 388, 154-170. [CrossRef]

40. Chong, U.P. Signal model-based fault detection and diagnosis for induction motors using features of vibration signal in two-dimension domain. Stroj. Vestn. J. Mech. Eng. 2011, 57, 655-666. 
41. Xu, G.; Liu, M.; Jiang, Z.; Söffker, D.; Shen, W. Bearing fault diagnosis method based on deep convolutional neural network and random forest ensemble learning. Sensors 2019, 19, 1088. [CrossRef] [PubMed]

42. Lu, C.; Wang, Y.; Ragulskis, M.; Cheng, Y. Fault diagnosis for rotating machinery: A method based on image processing. PLoS ONE 2016, 11, e0164111. [CrossRef]

43. Kang, M.; Kim, J.M. Reliable fault diagnosis of multiple induction motor defects using a 2-D representation of Shannon wavelets. IEEE Trans. Magn. 2014, 50,1-13. [CrossRef]

44. Huang, N.E.; Shen, Z.; Long, S.R.; Wu, M.C.; Shih, H.H.; Zheng, Q.; Yen, N.-C.; Tung, C.C.; Liu, H.H. The empirical mode decomposition and the Hilbert spectrum for nonlinear and non-stationary time series analysis. Proc. R. Soc. Lond. Ser. A Math. Phys. Eng. Sci. 1971, 454, 903-995. [CrossRef]

45. Torres, M.E.; Colominas, M.A.; Schlotthauer, G.; Flandrin, P. A complete ensemble empirical mode decomposition with adaptive noise. In Proceedings of the 2011 IEEE International Conference on Acoustics, Speech and Signal Processing (ICASSP), Prague, Czech Republic, 22-27 May 2011; pp. 4144-4147.

46. Daubechies, I. Ten Lectures on Wavelets; ser. CBMS-NSF Regional Conference Series in Applied Mathematics; SIAM: Philadelphia, PA, USA, 1992.

47. Jaffard, S.; Meyer, Y.; Ryan, R.D. Wavelets: Tools for Science and Technology; SIAM: Philadelphia, PA, USA, 2001.

48. Mallat, S. A Wavelet Tour of Signal Processing: The Sparse Way, 3rd ed.; Elsevier/Academic: New York, NY, USA, 2009.

49. Huang, S.J.; Hsieh, C.T. High-impedance fault detection utilizing a Morlet wavelet transform approach. IEEE Trans. Power Deliv. 1999, 14, 1401-1410. [CrossRef]

50. Lin, J.; Qu, L. Feature extraction based on morlet wavelet and its application for mechanical fault diagnosis. J. Sound Vib. 2000, 234, 135-148. [CrossRef]

51. Peng, Z.K.; Chu, F.L. Application of the wavelet transform in machine condition monitoring and fault diagnostics: A review with bibliography. Mech. Syst. Signal Process. 2004, 18, 199-221. [CrossRef]

52. Verstraete, D.; Ferrada, A.; Droguett, E.L.; Meruane, V.; Modarres, M. Deep Learning Enabled Fault Diagnosis Using Time-Frequency Image Analysis of Rolling Element Bearings. Shock Vib. 2017, 2017, 5067651. [CrossRef]

53. Image Processing in Python. Available online: http://scikit-image.org/docs/dev/auto_examples/transform/ plot_rescale.html (accessed on 12 May 2019).

54. Nwankpa, C.; Ijomah, W.; Gachagan, A.; Marshall, S. Activation Functions: Comparison of Trends in Practice and Research for Deep Learning. arXiv 2018, arXiv:1811.03378.

55. Krizhevsky, A.; Sutskever, I.; Hinton, G.E. ImageNet classification with deep convolutional neural networks. In Proceedings of the Conference on Neural Information Processing Systems (NIPS12), Lake Tahoe, NV, USA, 3-6 December 2012; pp. 1097-1105.

56. Xu, B.; Wang, N.; Chen, T.; Li, M. Empirical Evaluation of Rectified Activations in Convolutional Network. arXiv 2015, arXiv:1505.00853.

57. Park, S.; Kwak, N. Analysis on the Dropout Effect in Convolutional Neural Networks. In Computer Vision-ACCV 2016; Lecture Notes in Computer Science; Lai, S.H., Lepetit, V., Nishino, K., Sato, Y., Eds.; Springer: Cham, Switzerland, 2017; Volume 10112, pp. 189-204.

58. Kingma, D.P.; Ba, J. Adam: A method for stochastic optimization. arXiv 2014, arXiv:1412.6980.

59. Chang, H.; Kuo, C.; Hsueh, Y.; Wang, Y.; Hsieh, C. Fuzzy-Based Fault Diagnosis System for Induction Motors on Smart Grid Structures. In Proceedings of the IEEE International Conference on Smart Energy Grid Engineering, Oshawa, ON, Canada, 14-17 August 2017.

60. The Dataset Used for This Study. Available online: https://drive.google.com/drive/u/0/folders/1Cvs1LYagNmEQcj-s395dZflh3L4JWTO (accessed on 12 May 2019).

61. Zhang, X.; Liang, Y.; Zhou, J. A novel bearing fault diagnosis model integrated permutation entropy ensemble empirical mode decomposition and optimized SVM. Measurement 2015, 69, 164-179. [CrossRef]

62. Guo, X.; Chen, L.; Shen, C. Hierarchical adaptive deep convolution neural network and its application to bearing fault diagnosis. Measurement 2016, 93, 490-502. [CrossRef]

(C) 2019 by the authors. Licensee MDPI, Basel, Switzerland. This article is an open access article distributed under the terms and conditions of the Creative Commons Attribution (CC BY) license (http://creativecommons.org/licenses/by/4.0/). 